\title{
家庭的嬗变与重构一一新历史主义视域下的《如此世道》
}

\author{
高健 \\ 中国人民大学外国语学院 \\ DOI:10.32629/er.v3i2.2465
}

[摘要] 《如此世道》是英国王朝复辟时期威廉 - 康格里夫的代表作。这部作品以17世纪末的真实历史为背景, 通过描写一个上层阶级家庭中 发生的故事来映射现实社会在政治、文化、财产制度、思想观念等方面发生的变化,进而展现当时英国社会在急剧动荡时期的形态,并通过虚构 的情节来重构新的历史。本文就以新历史主义为视角, 着重研究作品中历史与文本的互动, 指出作品所体现的文本的历史性和历史的文本性。即 研究历史对作者的影响、《如此世道》所记述的历史事实以及《如此世道》中所构建的历史。

[关键词]《如此世道》; 家庭；新历史主义；文本的历史性；历史的文本性

\section{The Evolution and Reconstruction of the Family}

\section{Gao Jian}

\section{RUC.School of Foreign Languages}

[Abstract] The Way of the World is a masterpiece of William Congreve during the Restoration period. Based on the real history at the end of the 17th century, this work reflects the changes in politics, culture, property system, ideology and other aspects of the real society by describing the stories that happened in an upper class family, and then shows the status of the British society in the period of rapid turbulence at that time, and reconstructs the New History through fictional plots. From the perspective of new historicism, this paper focuses on the interaction between history and text in the works, pointing out the historicity of text and the textuality of history embodied in this work. That is, to study the influence of history on the author, the historical facts recorded in the book and the history constructed in the book. [Key word] The Way of the World; family; New Historicism;historicity of text; textuality of history

1700年, 威廉 - 康格里夫的《如此世道》在伦敦上映。这部作品继承 并发展了 17 世纪初期英国剧作家本 -琼森、马斯顿等人的社会讽刺喜剧传 统, 以讽刺和批判为主要写作目的 ${ }^{[8]}$ 。作为最后一部复辟时期的戏剧, 《如 此世道》并未获得当时观众的关注, 然而后世评论家们则广泛认为这是康 格里夫最好的作品, 是它赋予了风俗喜剧最后的光辉。

该作品描写了英国斯图亚特王朝复辟时期的家庭故事。一对青年男女 相爱, 但是两人的结合的过程中却夹杂了长辈的阻碍, 其他人的恋情以及 财产的争夺等一系列的事情。小说反映了复辟时期新旧力量的较量以及上 流社会的不良习气, 称得上是对复辟时期社会情况的辛辣讽刺和真实描 述。在王朝复辟以及接踵而来的光荣革命背景之下, 主流社会与历史都更加 关注社会大现象。本剧作将目光集中到社会最小的单元一一家庭上, 用家庭 中人物力量的此消彼长和观念的变化折射社会中正在发生的变革。本剧作 在上个世纪重回大众视野, 许多学者和评论家认为它能帮助了解复辟时期 历史的细枝末节。国外对本剧作的研究大多将其归在复辟时期戏剧与 18 世 纪戏剧对比的大框架之下。在上世纪 60 年代及 80 年代, 弗兰克 - 布拉迪和詹 姆斯 - 汤普森的研究都指出两个时期的戏剧创作水平以及受观众欢迎的程 度都是受政治转向影响 ${ }^{[1]}$ 。詹姆斯 - 汤普森在文中指出, 康格里夫最后一部 作品不受欢迎很大程度与此有关 ${ }^{[4]}$ 。理查德 - 布雷弗曼则谈论了剧本中体现 出的资本经济的发展是导致米拉贝尔胜于费纳尔的根本原因 ${ }^{[2]}$ 。还有罗伯 特 - 诺伊, 他着重研究了本剧在表演时将音乐效果运用于舞台的尝试 ${ }^{[3]}$ 。

而国内对于本剧的研究屈指可数, 且仅提出了爱情伦理和婚姻伦理两 个研究维度。本文将从新历史主义的视角出发, 研究作品中体现的文本和 历史的关系, 为日后的研究提供新的视野。

\section{1 新历史主义}

上世纪八十年, 斯蒂芬・格林布拉特第一次提出新历史主义。这是一种 以文化人类学的方式把整个文化当作研究对象的批评理论 ${ }^{[13]}$ 。这一定义明
确了自六十年代以来快速发展的与新批评主义、结构主义以及后结构主义 相对的文化研究方式。新历史主义者反对旧历史主义所持有的历史是真实 且客观的说法。新历史主义的代表人物海顿 - 怀特认为, 历史是一个文本, 是被研究和阐释的对象, 不存在绝对客观或真实的历史 ${ }^{[6]}$ 。此外, 在研究一部 作品时, 传统历史主义者常常希望能够重现作者在创造作品时的意图、世界 观和文化背景, 这就脱离了研究对象本身。基于此, 新历史主义者指出应当 深入了解文本中的社会存在及其反映的社会形态, 研究文本中的历史与社 会。新历史主义批评还纠正了文学反映论中单一和被动地倾向, 认为非历史 文本之间以及非历史文本与历史文本之间会产生互文 ${ }^{[7]}$ 。这些想法成为了新 历史主义者有关 “历史的文本性” 和 “文本的历史性” 的基础观点。代表人 物路易斯 - 孟酬士认为, 新历史主义批评的目的是重新描绘文本与塑造出文 本的文化现象之间的关系, 还要探讨文本中所反映的政治和意识形态要素。

本文就将从 “历史的文本性” 和 “文本的历史性” 这两个角度来分析 康格里夫的《如此世道》, 并探讨这部作为复辟时期落幕之作的风俗喜剧 与当时社会历史之间的关系。

\section{2 文本中所反映的真实历史}

新历史主义认为, 文本与历史之间具有互文性。文本是被压缩的历史, 历史是延伸的文本 ${ }^{[7]}$ 。根据格林巴特所说, 文学批评论及艺术作品与所反 映的现实之间的关系, 最常用的是摹仿。艺术作品摹仿的对象即为社会存 在的现实。孟酬士认为, 文学文本是历史语境的作用和表达 ${ }^{[13]}$ 。作家在进 行创作时是 “能动性” 与 “屈从性” 的统一体, 所创做出的作品即为作者 与社会协商的 “通货” ${ }^{55}$ 。也就是说文学文本在一定的历史环境中被创造 出来, 不可避免地受到历史环境的影响, 但是同时它又将历史环境反映在 文本之中。《如此世道》通过发生在家庭内的故事折射复辟时期社会上的 变化, 本章节就主要分析本部作品中所反映的一系列历史事件和现象。

2.1父权权威和君主制的衰落 
1660年, 查理二世复辟, 要求加强王权。然而16世纪下半叶, 资本主义经济 早已迅速发展, 日渐强大的资产阶级新贵族无法忍受封建君主的专制统治。但 是查理二世无视这些变化, 坚持 “国王的神圣权利”, 但是这种要求与当时社 会发展的现实要求不符。查理二世在统治时期的最后四年, 甚至从不开国会; 詹姆斯二世更是将自己的权力置于无上地位, 要求国会及国民 “无条件地服从 国王意志” ${ }^{[9]}$ 。在家庭中, 父亲就相当于社会中君主。父亲与君主居于各自领 域的主导地位。家长希望用命令形式来维持自己在家中的核心力量和绝对权 威。但从1500年到1700年间, 上层阶级的家庭中, 亲属关系(指除夫妻核心关系 之外的亲属关系) 、扈从关系影响力减弱 ${ }^{[12]}$, 家庭的核心人物 “父亲” 在对亲 属及扈从的管控上大不如前, 仅能在更小的范围内施加强有力的影响。根据英 国史学家劳伦斯 ・斯通的研究, “父权权威” 在17世纪之际已全面衰退。在《如 此世道》中, 康格里夫创造了一个旧权威、旧家长的代表人物: 威士福特夫人。

威士福特夫人是家长权威的代言人。作为塞妇, 她取代了同等地位的男 性角色, 拥有在家庭中的绝对权威。为了维护其权威, 威士福特夫人要求每个 人都遵循她的想法行事。开篇她便以一个愤怒、严厉的 “父亲” 形象出现在 男主角米拉贝尔和费纳尔的谈话中。她反对米拉贝尔向侄女米勒敏特的求婚, 仅仅是因为这个男人曾经得罪过自己。为了保证财产一直属于自己的家族, 她还准备将米勒敏特嫁给自己的侄子。“她 (米勒敏特) 的一半财产都要仰仗 姨妈认可的婚姻” ${ }^{[10]}$ 。可见威士福特夫人希望通过控侄女的财产来实现对其 婚姻的操纵并确保自己对她的绝对权威。然而, 她忽视了自己与米勒敏特的 关系绝对不是 “父亲” 与女儿的关系, 而仅仅是 “父亲” 的化身与非直系亲 属的关系。随着亲属关系的衰微, 她对米勒敏特的控制也难以为继。

此外, 即使是作为 “父亲”, 拥有父系权威, 威士福特夫人也完全没有 意识到自己已经进入家中其他成员的陷阱。米拉贝尔正筹划让已婚的仆人 佯装与自己合不来叔叔, 去威士福特夫人那里骗婚, 由此给她扣上与有夫 之妇结婚的罪名; 而女婿费纳尔在得知米拉贝尔的圈套后, 则和情人准备 将计就计, 等到真相大白时, 以费纳尔夫人和威士福特夫人的名誉为筹码, 要挟威士福特夫人把女儿的婚前财产都给他。

此时期家长的命运和国王的命运非常相似, 对旧有的从属力量难以把 控, 附属力量逐渐成长为新的势力, 最终使得旧力量难以与新力量抗衡。尽 管威士福特夫人希望通过家长权力来控制年轻人的自由选择,维护自己的 绝对权威, 但是年轻一代并未将古旧的家长权力放在眼里。大家长沦落为 小家长, 这正如君主权力逐渐不被资产阶级新贵重视一般。君主权力只能 在有限的范围内发挥作用。因此, 处于风雨飘摇中的家长和君主不得不向 新兴力量低头。威士福特夫人面临女婿勒索财产的危机时, 不得不以同意 侄女和米拉贝尔的亲事为好处, 请求他的帮助。君主则直接被资产阶级推 翻, 詹姆斯二世被驱逐出英格兰, 君主制也在光荣革命后成为君主立宪制, 君主再也没有实际的权力, 只作为国家象征而存在。

2.2 核心家庭模式的变化

17 世纪中叶, 随着亲属关系的逐渐衰微, 再加上 17 世纪初清教一派兴 起情感个人主义, 英国开始出现新的核心家庭。此类核心家庭强调夫妻之 间的深厚情感为婚姻的必需品 ${ }^{[12]}$ 。曾经盛行于 16 世纪末 17 世纪初的以婚姻 为商业的态度一去不复返。《如此世道》中, 康格里夫所刻画的两对情侣: 费纳尔夫妇和米氏情侣恰好体现了家庭模式转型前后的两个典型范例。

文中虽未直接言明费纳尔夫妇是如何结合的, 但是从费纳尔筹划从岳母 手里套钱的整个过程来看, 很明显费纳尔夫妇之间根本不存在情感, 有的只是 唾手可得的利益。费纳尔在与妻子的朋友玛伍德太太偷情回家后, 会口是心非 地对妻子表达爱意, 他会称呼妻子 “亲爱的” , “小宝贝” ${ }^{[10]}$ 。在与马伍德说 话时, 他直接道出了与妻子成婚的缘由: “要不是看中了这个寊妇未来的资产, 我干嘛和她结婚? 我干嘛还要把这个寡妇的财产浪费在爱情和你身上? ” ${ }^{[10]}$ 。
他甚至想要 “抢光她的财产”。可见, 费纳尔和妻子的婚姻纯粹建立在物质的 基础上, 毫无感情根基。费纳尔太太完全能感觉到丈夫的敷衍和庆恶, 她说: “我的丈夫。你没看到他么? 他下意识地不待见我, 快把我压得喘不过气了” [10]。同样的, 当马伍德问费纳尔太太, “你恨你的丈夫吗？” 时, 费纳尔夫人回 答: “恨之入骨, 没错, 恨到极致” ${ }^{[10]}$ 。费纳尔夫妇对他们的婚姻毫无依恋之感, 两人的婚姻名存实亡。这种不稳定的婚姻关系在国家层面而言, 是一种极不稳 定的因素, 因此宗教在宣传新型家庭关系开始发挥越来越大的作用。

实际上, 17 世纪新教在教民之中宣传 “神圣婚姻” 的力度加大, 认为以婚 姻为买卖的行为违反了教义。所以当费纳尔内心算计自己的妻子时, 马伍德 “善意” 提醒费纳尔: “婚姻所缔, 神圣无欺” ${ }^{[10]}$ 。这种婚姻神圣观对当时社 会各阶级中产生了深远普遍的影响。再加上情感个人主义在家庭中的觉醒, 要求维系家庭稳定的因素为夫妻情感, 新的核心家庭开始一步步形成。尽管 剧本中另一对情侣米拉贝尔和米勒敏特对于财产的仍有一定的要求, 但是二 人相比于费纳尔夫妇更加强调情感为婚姻的基础。米拉贝尔对于米勒敏特的 爱溢于言表。当与费纳尔谈及米勒敏特时, 米拉贝尔感叹: “作为一个敏感又 热烈的情人, 我爱他连同她的缺点一起爱; 不, 我因为她的缺点而爱她” ${ }^{[10]}$ 。 相比之下, 米勒敏特还是拥有着 17 世纪女性含蓄内敛的性格, 不敢直接回应 爱人。但是当马伍德说自己讨厌米拉贝尔时, 她会不留情面地回击: “奥, 太 太, 怎么说呢, 我也讨厌他。但是禁不住人家爱着我啊, 哈! 哈! 哈!” ${ }^{[10]}$ 。从 她讽刺马伍德对米拉贝尔爱而不得的话语中, 可见她十分满意自己爱的人也 爱着自己。此外, 米勒敏特还有着刚刚陷入爱情的迷茫和差涩, 尽管她在米拉 贝尔面前装作一副高冷的样子, 而且会在米拉贝尔说 “我爱着你” 时, 大叫别 说那个字, 但是这些举止都难以掩饰她也爱上了米拉贝尔。之后, 两人瞒着威 士福特夫人私定终身, 甚至请来了威士福特夫人的女儿作为婚约见证人。

两人的结合基于双方的互相爱慕, 日后的家庭也就将建立于这种情感 之上。除此之外, 里这对年轻情侣乃至婚约见证人费纳尔夫人都不曾想过要 将这件事告知威士福特夫, 这点显示出三人倾向于拥有自主选择情感和婚 姻的自由, 并未将家长身份放在高于其自身意愿的地位上。康格里夫对这对 情侣的由爱情走向定下婚约的描述, 不仅反映出社会对于以夫妻情感为基 础的新型核心家庭的形成与构建, 又再一次印证了家庭中亲属关系的衰微。

\section{3 作为文本的历史}

新历史主义者提出 “历史的文本性”, 用以表示历史实际上就是文本 的性质。在新历史主义者看来, 对特定时期的历史书写会受到笔者所处的 时代环境、话语环境的制约, 同时, 历史的记录者也会因个人偏见对历史加 以选择和评价。因此不存在真正客观和真实的历史, 历史和其他文学文本 一样, 被作者创造出来, 又被他人加以解读和阐释 ${ }^{[6]}$ 。所以历史也是虚构的 文本, 并且会对所处时代有重大影响。一般来说, 这种影响都会有利于统治 阶级统治, 或是引起被统治阶级的反抗。《如此世道》通过对资产阶级虚假 道德的隐晦描述, 以及对女性家庭地位的虚构都重新阐释和构建了历史。

3.1 “完美的” 资产阶级道德

新历史主义者认为, 历史作为由人根据自己的偏好创作的文本, 受到 了当时话语力量的影响, 具有维护统治阶级统治的作用。1688年光荣革命 可以视为资产阶级在英国的最大胜利, 成功成为国家和历史的主宰者。这 一时期的话语体系由资产阶级控制, 所谓官方的历史记录也将资产阶级放 在道德、能力、先进性等等方面的无上地位。然而, 新历史主义者强调要 考察历史中被边缘化的文本, 寻找主流话语所忽视的问题。

《如此世道》在 1700 年于伦敦上映时, 好评胗胗并未激起水花。同时代 的杰瑞米 - 科利尔还对其在剧作中的无道德书写专门写文章攻击, 甚至引发 了戏剧改革运动。光荣革命之后, 资产阶级要求为自己正名从而更加名正言 顺地管理国家事务, 并从中获益。这一时期内, 人民对于戏剧审美也发生了 
变化, 复辟前期的反映无道德的剧目无法吸引观众。这种审美的转变以及对 康格里夫作品的不接受恰恰说明了资产阶级统治在初期的胜利。因为《如 此世道》不仅通过讲述费纳尔与妻子的朋友偷情甚至想窃取妻子财产和情 人私奔的故事来讽刺复辟时期浪荡公子哥的道德败坏, 还以米拉贝尔为例 揭示了掩藏在资产阶级书写的历史下所谓的 “完美” 资产阶级道德。

米拉贝尔作为新力量、新阶级的代言人, 凭借自己的性格和智慧不仅赢得 了几乎所有女性的青睐, 反祄出费纳尔的无知, 还通过帮助威士福特夫人保护 财产获得了与她侄女结婚的许可。相比于费纳尔, 他在全心全意对待自己的爱 人, 乃至爱上爱人的缺点。从这些行为来看, 米拉贝尔似乎确实是一个对爱忠 贞、乐于助人的资产阶级上流人士的形象。然而, 若是对他在剧中的其他行为 或通过他人话语体现出的行为进行研究, 则能对他有个更加全面的了解。

米拉贝尔之前也是一个花花公子, 与费纳尔太太有染, 与马伍德太太关 系暖昧。费纳尔太太抱怨: “你那时就不能给你的恨设个限么? 你为什么要 让我嫁给他? ” ${ }^{[10]}$ 。可见米拉贝尔曾在费纳尔太太单身时与之相处, 但是分 手时出于恨意便建议她嫁给浪荡的费纳尔。而在费纳尔太太婚后, 米拉贝尔 仍与她关系不一般, 以至于费纳尔都有所察觉 “我夫人和他眉来眼去, 我视而 不见” ${ }^{[10]}$ 。因此, 米拉贝尔对于爱情和婚姻能否保持持久的忠贞还是未知数。 同时, 为了能与爱人结婚, 米拉贝尔要求自己的仆人先与威士福特夫人身边 的侍女结婚, 然后让他假装是想向自己复仇的叔叔去骗取威士福特夫人的信 任再与她结婚。这样一来, 威士福特夫人犯下的就是让人重婚的罪行。这时 米拉贝尔就可以以这件丑事为筹码要挟威士福特夫人答应自己与米勒敏特 的婚事。这个计划天衣无缝, 并且一旦成功执行就十分有效。但是, 米拉贝尔 以自己仆人的婚恋自由为代价, 换取自己的婚恋自由。他并没有做到资产阶 级宪法中 “人人平等” 的规定。此外, 在算计爱人的姨妈的过程中, 米拉贝尔 也没有考虑过这个计划可能会对爱人产生的影响: 如果成功, 米勒敏特将把 自己的爱情与婚姻建立在姨妈的痛苦之上; 如果失败, 姨妈将会怎样对待米 勒敏特。资产阶级的虚伪与自私自利的本性在这个过程中暴露无遗。

可以说, 康格里夫的这部剧作中不存在真正完美的形象, 即使是当时 被政府宣传的极为正面的资产阶级, 也是有着花花肠子和虚伪自私本性的 人。剧中人物的刻画与历史记录之间的出入恰好说明了历史也不过是包含 着虚构因素的文学文本, 历史也只是另一种以一层次上的 “历史修撰” “11] 《如此世道》作为当时不被人重视的剧目实际上为后世对当时历史文本的 研究提供了新的视角和阐释。

3.2女性家庭地位的提升

在剧本中, 米勒敏特以一个平等意识觉醒的女性出现。当米拉贝尔与 米勒敏特私下见面时, 米勒敏特正在犹豫是否要接受米拉贝尔的求婚。她 向这个对自己痴心的男人倾诉了自己对于婚姻的焦虑和恐慌, 并提出了数 条婚后的规章。毕竟 “自由如此宝贵, 我该抛下它么? ” ${ }^{[10]}$ 。对米拉贝尔 的发问暗含着米勒敏特对于婚后自由与权力的思考。

最让米勒敏特感到忧虑的就是名字问题。她对于婚后自己的名字将要 发生改变感到十分痛心。“妻子, 配偶, 挚爱, 欢乐的源泉” ${ }^{[10]}$ 等等称号, 都 将取代 “米勒敏特”。这是米勒敏特自我意识的体现。在父权制社会中, 妇女失去了发言权, 被视为男性的附属物。剥夺女性的名字正是剥夺了她 们的独立地位与话语权。因此, 名字是女性展现自我并让她们了解自己独 立身份的最重要的要素。但是宗教和社会制度使妇女处于劣势。《圣经》 将夏娃的诞生视作建立在男性的牺牲之上, 女人自始至终不过是男人身上 的一条肋骨, 是男人的附属物。米勒敏特想要在婚后维持自己的独立身份, 依旧保有自己的名字。因此她向米拉贝尔要求 “我决不允许被冠以这样的 名字 (代称) ” ${ }^{[10]}$ 。除此之外, 米勒敏特还要求拥有与米拉贝尔一样的与人 交往、拥有私人空间的权利。米拉贝尔接受了爱人所有的要求, 并认为这
些要求是合理的。他的态度非常重要, 因为他是父权中的一员, 在某种程度 上, 他的想法代表了其他男性的想法。他的态度可以解读为男性整体对于 女性在家庭中享有与自己平等权利和自由的认可。

然而, 虽然 17 世纪新的核心家庭是建立在夫妻情感的基础之上, 但这 并不意味着夫妻关系的平等。中上阶级的妇人在家中的地位依旧处于劣势, 男性是她们的统治者。妻子有义务要 “服从、敬侍丈夫” ${ }^{[12]}$ 。在家庭中, 女性依旧处于劣势地位, 可能有个别的男性会允许女性有与自己平等的 权力, 但是当时的大部分家庭之中依旧是男尊女卑的格局。尽管 17 世纪 末期出现了女性主义运动, 但是其中大半流产, 且对公众的态度没有很 大影响 ${ }^{[12]}$ 。因此, 康格里夫在剧中不遗余力地书写米拉贝尔与米勒敏特在 关于婚姻关系中地位的对话, 以及米拉贝尔毫不犹豫地答应爱人请求并认 为合理的态度, 实际上是他对于实现女性家庭地位平等的虚构和期盼。

很难说这部上映与 18 世纪元年的戏剧改变了大众对于女性地位的看 法, 但是有关女性权利与地位的问题无疑再次被挑起。发生在18世纪的女 性主义浪潮很有可能受到了前期女性运动以及类似《如此世道》的戏剧的 影响, 进而去影响和书写历史。

\section{4 结语}

本文以新历史主义为视角, 围绕着《如此世道》里家庭中的父权、家 庭核心、男女道德以及女性家庭地位四个方面展开讨论, 对《如此世道》 与当时历史的关系进行了解读。

作为复辟时期的一部剧作，《如此世道》是康格里夫在当时历史情境 下与社会协商的产物。从该文本与社会、其他文本的互文中可以看出历史 情境施加在作者及作品上的影响。可以说, 《如此世道》既是历史语境下 生成的文本, 也在解读和虚构着历史。剧作中反映和描绘复辟时期君权、 家长权威的衰落, 以及核心家庭的转变, 让观众和读者能够更加了解复辟 时期英国从大社会群体到家庭这个小社会群体的大致面貌。而剧作对历史 记录中资产阶级 “完美” 道德给出完全相反的阐释, 则为历史文本的研究 提供了另一种可能。文本还展示了在当时社会情境下虚构出的女性平等地 位, 表现了作者对于历史的重构的书写。读者和观众可从阅读及观看本剧 的同时, 感受历史与文本之间的碰撞和互文, 并构建别样的历史文本。

\section{[参考文献]}

[1]Brady,Frank.Recent Studies in the Restoration and Eighteenth Century[J].Studies in English Literature,1500-1900,1968,8(3):55-572.

[2]Braverman,Richard. Capital Relations and The Way of the World [J].ELH,1985,52(1):133-158.

[3]Noyes,Robert Gale. Songs from Restoration Drama in Contemporary and Eighteenth-Century Poetical Miscellanies[J].ELH,1936,3(4):291-316.

[4]Thompson,James.HISTORIES OF RESTORATION DRAMA[J].The Eighteenth Century,1983,24(2):163-172.

[5]陈澄.文本的历史性和历史的文本性[D].南京师范大学,2007.

[6]陈世丹.当代西方文艺批评理论要义[M]. 北京: 中国人民大学出版社,2018.

[7]丁佩.从新历史主义角度解析《愤怒的葡萄》[J].湖北经济学院学 报(人文社会科学版),2018,15(01):88-90.

[8]何其莘.王朝复辟时期的风俗喜剧[J].外国文学,1998,(05):63-71. [9]屈勒味林,钱端升译.英国史 [M].北京:红旗出版社,2017.

[10][英]威廉・康格里夫,叶佳煌,夏菁.如此世道[M].译言・古登堡计划,2019. [11]盛宁.文学・文论・文化 $[M]$. 济南: 山东友谊出版社,2006。

[12]劳伦斯 - 斯通, 刀篠华译.英国的家庭、性与婚姻1500-1800[M]. 北京:商务印书馆,2011.

[13]张京媛.新历史主义与文学批评[M].北京: 北京大学出版社,1993. 